\title{
Recurrent spontaneous coronary artery dissection: A case report and review of the literature
}

\author{
Jacqueline L Harris MBBS ${ }^{1}$, John Brereton MBBS FRACS ${ }^{2}$, Cathy S Lim BScMed MBBS MPH ${ }^{3}$, \\ Gregory IC Nelson MBBS FRACP ${ }^{1}$
}

\begin{abstract}
JL Harris, J Brereton, CS Lim, GIC Nelson. Recurrent spontaneous coronary artery dissection: A case report and review of the literature. Int J Angiol 2007;16(3):109-112.
\end{abstract}

Spontaneous coronary artery dissection is a rare cause of acute coronary syndrome. Recurrent spontaneous dissection is even more rare.

\section{CASE PRESENTATION}

A female patient first presented to her local rural hospital complaining of chest pain at 30 years of age. Her medical history revealed four uncomplicated pregnancies, recurrent migraines and repair of a childhood branchial cyst. She had normal blood pressure and cholesterol, and was an ex-smoker of five packyears. Her family history was remarkable for her mother dying in her fifties of acute myocardial infarction soon after an aortic valve replacement. An examination revealed hypermobile joints, but no other features to suggest Marfan's syndrome. An electrocardiogram (ECG) at the time of presentation revealed anterolateral ST segment elevation consistent with acute myocardial infarction. The patient was treated initially with thrombolysis, in addition to intravenous heparin, nitrate therapy, beta-blockade, morphine and acetylsalicylic acid. The patient complained of persistent pain and was transferred to a tertiary care hospital for ongoing management. Angiography revealed diffuse $75 \%$ narrowing of the left main coronary artery, with total occlusion of the left anterior descending artery (LAD) at its origin and critical obstruction of the left circumflex artery. The dominant right coronary artery (RCA) was normal. The left ventricle was dilated with moderately severe anterolateral hypokinesis. The appearances were suggestive of coronary artery dissection (Figures $1 \mathrm{~A}$ and $1 \mathrm{~B}$ )

Consequently, the patient underwent coronary artery bypass surgery and dissection was confirmed. The anterior myocardium was thin, suggesting an older subclinical myocardial infarction. Operative inspection revealed no evidence of atheromatous disease within the aorta or coronary arteries. Bilateral internal mammary artery grafting was attempted; however, these arteries spontaneously dissected and peripheral vein grafts were used. Histopathology of the internal mammary arteries showed cystic medial necrosis (Figure 2). Postoperative recovery was uncomplicated.

The patient presented again five years later with a further episode of chest pain. An ECG revealed inferior ST segment elevation consistent with acute myocardial infarction, and the patient was transferred to a tertiary care hospital without the administration of thrombolysis. Angiography demonstrated a
A case of recurrent coronary artery dissection is reported and the literature is reviewed.

Key Words: Acute myocardial infarction; Cystic medial necrosis; Spontaneous coronary artery dissection

long dissection of the dominant RCA with total occlusion of the posterior descending artery (Figure 3). All vein grafts were patent, and the left main coronary artery and LAD were now normal (Figure 4). Left ventriculography showed a slightly dilated left ventricle with mild anterolateral and moderate apical hypokinesis; however, there was improvement in systolic function when compared with the original consultation. The patient again underwent coronary artery bypass grafting, which was uncomplicated.

Three years subsequent to this, the patient again presented with chest pain. An ECG revealed no acute changes; however, serial troponin T peaked at $0.14 \mu \mathrm{g} / \mathrm{L}$. Angiography revealed residual subtotal occlusion of the right coronary artery with no new changes. Telemetry during this admission revealed recurrent asymptomatic nonsustained ventricular tachycardia. Electrophysiological studies were normal. Aortic dissection was excluded with computed tomography aortography, and an implantable defibrillator was inserted. The patient was discharged home, and review in a specialized Marfan clinic was arranged. Continuation of beta-blocker therapy and avoidance of high-risk contact sports were advised for the patient and her children. Genetic testing has not been recommended.

\section{DISCUSSION}

Spontaneous coronary artery dissection (SCAD) is a rare cause of acute coronary syndrome (1) and is described as dissection of the coronary arteries without a known precipitant. Since the first case of SCAD was described by Pretty in 1931 (2), there have been over 266 cases reported in the literature (3).

SCAD can present along any point of the acute coronary syndrome spectrum. The incidence of SCAD is unknown and is probably underestimated in the literature. In a recent review of 5054 consecutive coronary angiograms in Switzerland, the incidence of idiopathic SCAD was $0.1 \%$ (4); however, the incidence has been reported to be up to $1.1 \%$ (5). Of these cases, the incidence was highest among those patients presenting with unstable angina pectoris or postinfarction angina $(4.2 \%)$, and acute myocardial infarction $(2.9 \%)$, compared with stable angina pectoris $(0.3 \%)(5)$. There is a

${ }^{1}$ Departments of Cardiology, ${ }^{2}$ Cardiothoracic Surgery and ${ }^{3}$ Pathology, Royal North Shore Hospital, Sydney, Australia

Correspondence: Jacqueline L Harris, 18a Berryman Street, North Ryde 2112, Australia. Telephone 61-2-98891176, fax 61-2-403490672,

e-mail jacq@exemail.com.au 

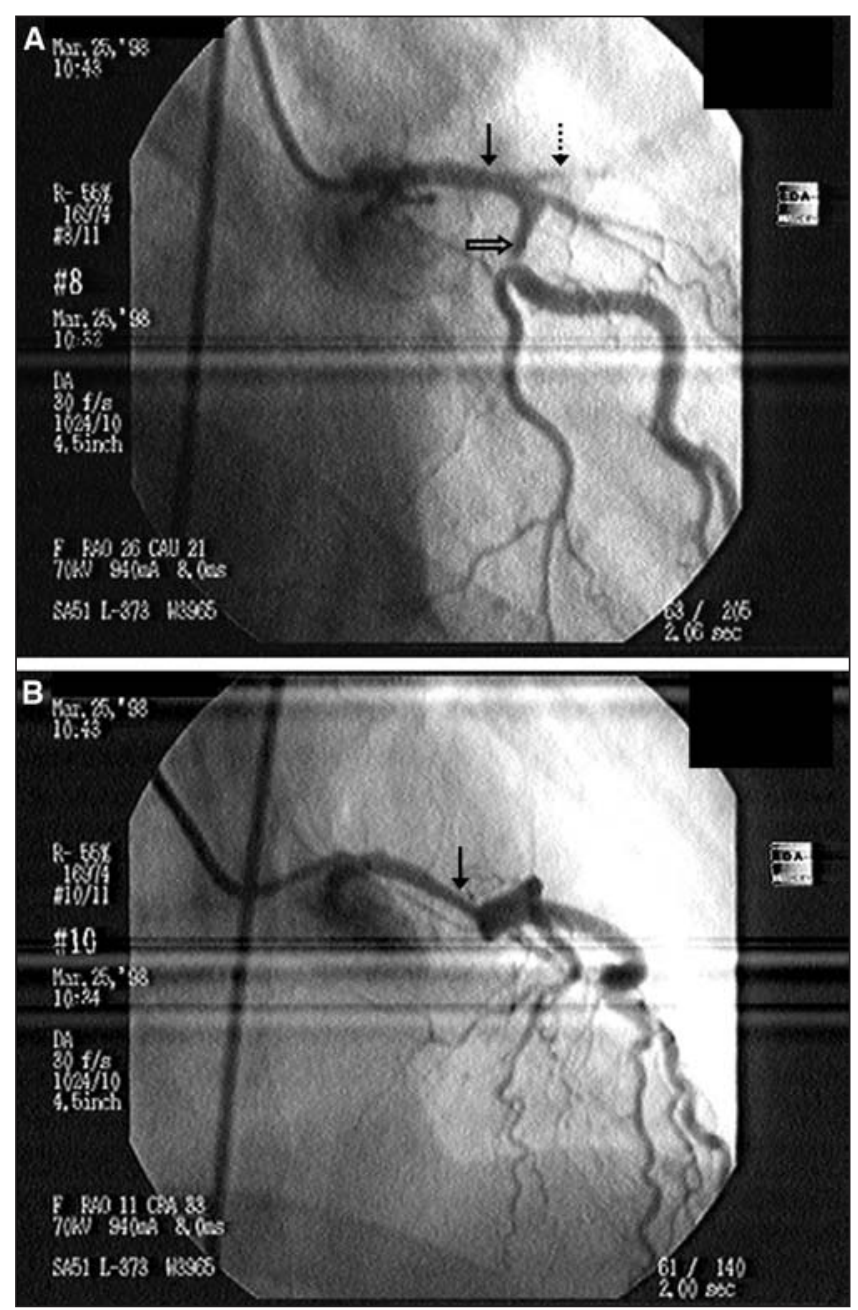

Figure 1) Coronary angiogram showing (A) left main coronary artery narrowing (solid arrow), complete proximal obstruction of the left anterior descending artery (dotted arrow) and narrowing of the left circumflex artery (outlined arrow). (B) Left main coronary artery narrowing (solid arrow)

strong predominance in women ( $63 \%$ to $80 \%$ of cases), with a mean age at presentation of 35 to 46 years $(6,7)$.

There are three main subgroups of patients who present with coronary artery dissection (8). The first group of patients includes those with underlying atherosclerosis; it accounts for $45 \%$ of cases. The second group includes patients whose arteries dissect in association with pregnancy or the postpartum state, which accounts for $12 \%$ of cases. The third group includes those without any identifiable risk factors and accounts for $34 \%$ of cases (6). Our patient falls into this 'idiopathic' group.

The pathophysiology of SCAD is poorly understood. This is because it is a rare condition, but also because the pathophysiology is probably heterogenous. The etiology in the patient group with underlying atherosclerosis is presumed to be secondary to increased density of vasa vasorum and intimal injury leading to intramural hemorrhage and subsequent dissection (9). The 'idiopathic' patient group probably represents different connective tissue disorders or vasculitides (10) presenting with a common clinical syndrome. Our patient's history supports this theory because cystic medial necrosis is associated with connective tissue disorders. It is
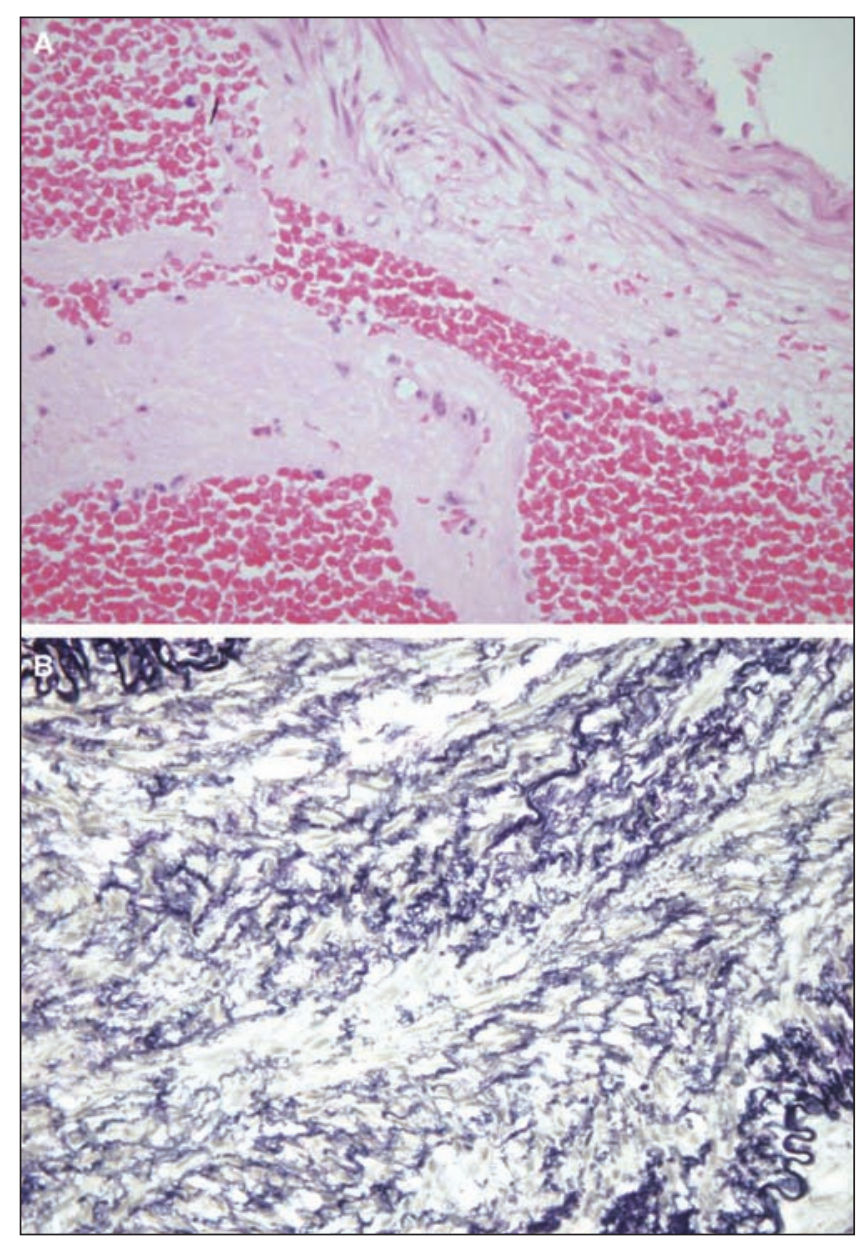

Figure 2) Histopathology of the internal mammary arteries showing cystic medial necrosis. Dissection through the media of the mammary artery (a) (hematoxylin and eosin stain, $\times 200$ magnification). Fragmentation and reduplication of the elastic tissue (B) (Verhoeff van Giesen stain, $\times 400$ magnification)

commonly associated with spontaneous aortic dissection, although our patient has no evidence of aortic disease. Cystic medial necrosis was reported to be the cause of SCAD in only a few cases (3). It is thought to have a genetic basis and is often autosomal dominant; hence, our patient's mother's history is suspicious for dissection. This also has implications for her children. The pregnancy-associated group is thought to have a predisposition to dissection secondary to hemodynamic and structural vascular changes related to hormonal influences $(1,4)$. They may also have an underlying connective tissue disorder with the added predisposition from pregnancy although, interestingly, our patient had four uncomplicated pregnancies.

The pattern of arterial involvement is similar to that of atherosclerotic disease, with $66 \%$ of cases affecting the LAD, $25 \%$ affecting RCA and $7 \%$ affecting the the left circumflex artery. Less than $10 \%$ of cases report multivessel dissection and/or left main coronary artery involvement (6). Men more commonly have dissection in the right coronary circulation (8).

The overall prognosis of SCAD is unknown because the groups and literature reviews are heterogenous and treatment options vary. Initial reviews of SCAD reported a mortality rate of $70 \%$ (11). More recently, the outcome of SCAD has been 


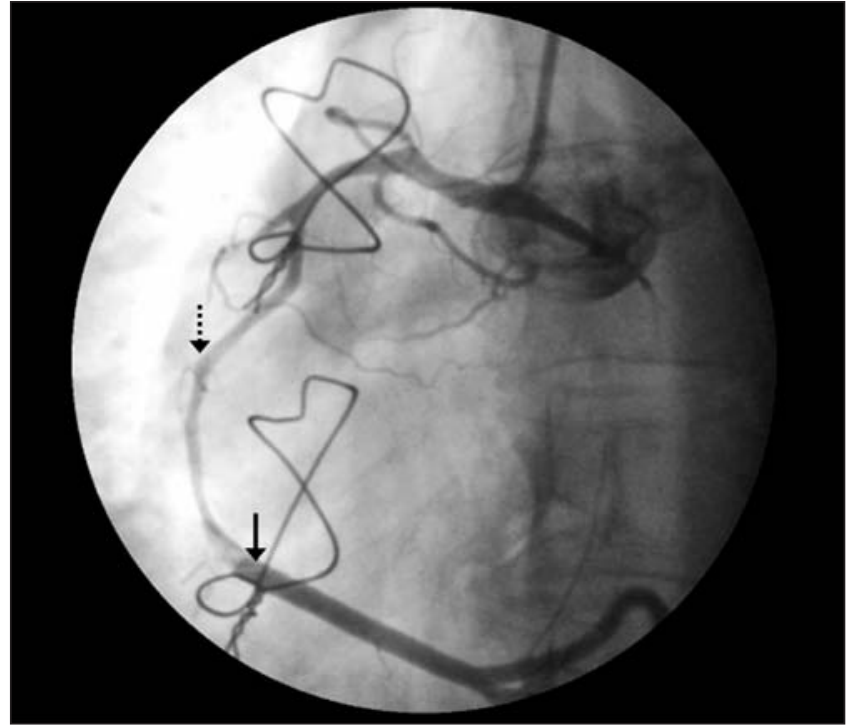

Figure 3) Right coronary artery dissection (dotted arrow) with dissection flap seen distally (solid arrow)

reported to be more favourable $(6,12)$, with one review suggesting a survival rate of $82 \%$ (8). Our patient's outcome has been remarkably good.

A management strategy of SCAD has recently been published based on the current literature (6). In patients with SCAD who do not have ongoing symptoms or hemodynamic instability, medical management (antiplatelet therapy, heparin and nitrate therapy; beta-blockade and glycoprotein IIb/IIIa inhibition) is recommended. All of these therapies were reported to be successful in case reports and case series. However, more substantial evidence from controlled studies is lacking. The use of thrombolytics is controversial, with both a theoretical and reported risk of increasing the propagation of dissection (13). In patients with ongoing ischemia or hemodynamic instability with single vessel SCAD, percutaneous coronary angioplasty and stenting is recommended. In patients with multivessel disease or left main coronary artery disease, or patients unresponsive to other options, coronary artery bypass grafting is recommended if feasible, or transplantation if not feasible. Immunosuppressive prednisolone and cyclophosphamide therapy has been reported as a successful treatment option in one peripartum woman with inoperable disease (14). Our patient's treatment supports these guidelines.

Our patient's case is also interesting as a documentation of significant recurrent disease. She had at least three separate events. The incidence of recurrent SCAD is unknown. In a retrospective review of SCAD associated with pregnancy, up to $20 \%$ of patients who survived the initial event had evidence of recurrent SCAD at follow-up (14). The recurrence of SCAD in patients other than peripartum women was first reported in 1982, in a postmortem case of a 42-year-old man (15). A recent case report of a 45-year-old woman with recurrent SCAD involving different coronary arteries two years apart is only the second case report of SCAD recurrence in the idiopathic patient group (16). The present case report is the third such case.

The literature on SCAD is growing; however, there is still a lack of understanding regarding the pathophysiology, prognosis and optimal treatment options for this rare disease. Our case

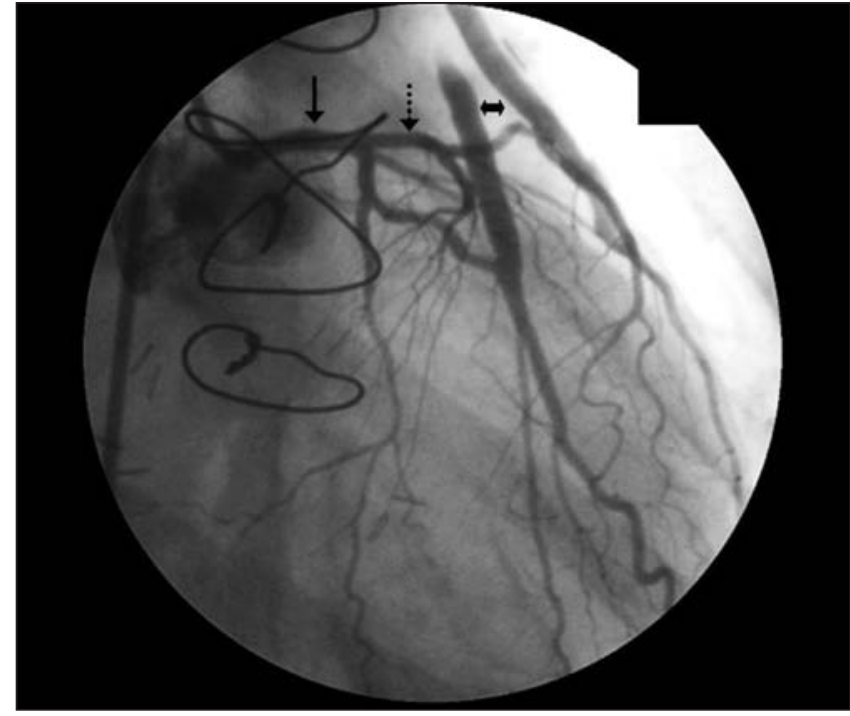

Figure 4) Coronary angiogram showing patent vein grafts to the left anterior descending artery (LAD) and left circumflex artery (doubleended arrow), resolution of dissection of the left main coronary artery (solid arrow) and resolution of complete obstruction of the LAD (dotted arrow)

report of a middle-aged woman with SCAD is important for a number of reasons, including the pattern of artery involvement and support for management options. Most importantly, it documents significant recurrence of disease despite risk factor management. It also gives pathological evidence for cystic medial necrosis as an underlying etiology without evidence of larger vessel involvement. It supports the idea that at least some of these patients have a connective tissue disorder with significant implications for relatives.

\section{REFERENCES}

1. Al-Daraji W, Howat AJ. Spontaneous coronary artery dissection. Histopathology 2005;47:215-6.

2. Pretty H. Dissecting aneurysm of coronary artery in a woman aged 42. BMJ 1931;1:667.

3. Kamineni R, Sadhu A, Alpert JS. Spontaneous coronary artery dissection: Report of two cases and a 50-year review of the literature. Cardiol Rev 2002;10:279-84.

4. Maeder M, Ammann P, Angehrn W, et al. Idiopathic spontaneous coronary artery dissection: Incidence, diagnosis and treatment. Int J Cardiol 2005;101:363-9.

5. Hering D, Piper C, Hohmann C, Schultheiss HP, Horstkotte D. [Prospective study of the incidence, pathogenesis and therapy of spontaneous, by coronary angiography diagnosed coronary artery dissection.] Z Kardiol 1998;87:961-70.

6. Verma PK, Sandhu MS, Mittal BR, et al. Large spontaneous coronary artery dissections - a study of three cases, literature review, and possible therapeutic strategies. Angiology 2004;55:309-18.

7. Dhawan R, Singh G, Fesniak H. Spontaneous coronary artery dissection: The clinical spectrum. Angiology 2002;53:89-93.

8. DeMaio J, Samuel J, Kinsella SH, Silverman ME. Clinical course and long-term prognosis of spontaneous coronary artery dissection. Am J Cardiol 1989;64:471-4.

9. Celik SK, Sagcan A, Altintig A, et al. Primary spontaneous coronary artery dissections in atherosclerotic patients. Report of nine cases with review of the pertinent literature. Eur J Cardiothorac Surg 2001;20:573-6.

10. Auer J, Punzengruber C, Berent R, et al. Spontaneous coronary artery dissection involving the left main stem: Assessment by intravascular ultrasound. Heart 2004;90:39-40. 
11. Robinowitz M, Virmani R, McAllister HAJ. Spontaneous coronary artery dissection and eosinophilic inflammation: A cause and effect relationship? Am J Med 1982;72:923-8.

12. Leone F, Macchiusi A, Ricci R, et al. Acute myocardial infarction from spontaneous coronary artery dissection: A case report and review of the literature. Cardiol Rev 2004:12:3-9.

13. Zupan I, Noc M, Trinkaus D, Popovic M. Double vessel extension of spontaneous left main coronary artery dissection in young women treated with thrombolytics. Catheter Cardiovasc Interv 2001;52:226-30.

14. Koller PT, Cliffe CM, Ridley DJ. Immunosuppressive therapy for peripartum-type spontaneous coronary artery dissection: Case report and review. Clin Cardiol 1998;21:40-6.

15. van der Bel-Kahn J. Recurrent primary coronary artery dissecting aneurysm. Am J Clin Pathol 1982;78:394-8.

16. Eddinger J, Dietz WA. Recurrent spontaneous coronary artery dissection. Catheter Cardiovasc Interv 2005;66:566-9. 